\title{
Matorral de "Mata Negra" (Mulguraea tridens [Lag.] N. O'Learly \& P. Peralta): una asociación vegetal amenazada por incendios en el Parque Nacional Torres del Paine, Chile
}

\author{
"Mata Negra" (Mulguraea tridens [Lag.] N. O'Learly \& P. Peralta) shrublands: \\ a plant community threatened by fires in Torres del Paine National Park, Chile
}

Osvaldo Vidal ${ }^{1}$, Carlos Ramírez ${ }^{2}$, Javier Latorre ${ }^{1}$,

Juan Marcos Henríquez ${ }^{1} \&$ Cristina San Martín ${ }^{3}$

\section{Resumen}

El Parque Nacional Torres del Paine (TDP) es uno de los escenarios naturales más importantes en Chile. La variabilidad topográfica y climática que imprime la Cordillera Paine, genera gradientes ambientales en donde es posible observar una vegetación representativa de la Ecorregión Magallánica Subantártica. Sin embargo, esta vegetación no ha sido suficientemente descrita, faltando por conocer muchos atributos de composición y estructura. Más aún, la vegetación en TDP está en peligro debido a los efectos de los últimos mega-incendios, que generan pérdida de hábitat, homogenización biótica e invasiones biológicas. Se describen atributos estructurales y de composición del matorral de Mata Negra (Mulguraea tridens), una comunidad dominada por esta especie con problemas de conservación, no descrita en detalle previamente. Se muestreó con metodología fitosociológica para caracterizar la diversidad florística del matorral. Se registraron 68 especies, siendo las más importantes Mulguraea tridens, Mulinum spinosum (Cav.) Pers. y Senecio patagonicus Hook. et Arn. El matorral está dominado por especies autóctonas, aunque las especies alóctonas son también importantes, denotando un incipiente proceso de invasión. La composición de especies de este matorral difiere a la registrada en zonas más septentrionales, indicando su particular identidad, razón por la que se le denomina Acaeno-Mulgaraetum tridentis. Los lignotuber de Mulguraea tridens tienen una baja tasa de rebrote post-fuego, por lo que es una comunidad sensible a los incendios, y la regeneración por semillas es crucial para su recuperación natural post-perturbación. Sugerimos que esta información puede ser útil para los manejadores del parque nacional, permitiéndoles incorporar información ausente sobre biodiversidad a los programas de manejo, y generar estrategias de restauración ecológica post-fuego para este matorral.

\section{Palabras clave:}

Mulguraea tridens, fuego, fitosociología, vegetación, Torres del Paine, restauración ecológica.

\section{Abstract}

The Torres del Paine National Park (TDP) is one of the most important natural landscapes in Chile. The topographic and climatic variability of Cordillera Paine, generates environmental gradients with typical vegetation of the Sub-Antarctic Magellanic Ecoregion. However, structural and

\section{Laboratorio de Botánica, Instituto de la Patagonia, Universidad de Magallanes. Avenida Manuel Bulnes 01890, Punta Arenas, Chile. osvaldo.vidal@me.com $\mathcal{R}$, javierlatorreb@gmail.com y jmarcoshenriquez@gmail.com}

2 Departamento de Ecología, Facultad de Ciencias Biológicas, Pontificia Universidad Católica de Chile. Alameda 340, 3er Piso Of. 320, Santiago, Chile. cramirez@uach.cl

3 Instituto de Ciencias Ambientales \& Evolutivas, Facultad de Ciencias, Universidad Austral de Chile. Campus Teja, Edificio Emilio Pugin, Valdivia, Chile. csanmart@uach.cl 
compositional attributes of this vegetation have not been described sufficiently. Moreover, TDP vegetation is endangered because of the effects of recent mega-fires, which generate habitat loss, biological invasions and biotic homogenization. Here, the structure and composition of Mata Negra (Mulguraea tridens) scrub attributes are described, a plant community dominated by this species with conservation problems not described in detail previously. The plants were sampled with phytosociological methodology to characterize the floristic diversity of the bush. Sixty-eight species were recorded, being the most important Mulguraea tridens, Mulinum spinosum (Cav.) Pers. and Senecio patagonicus Hook et. Arn. The scrub is dominated by native species, and even exotic species, indicating an incipient process of invasion. The composition of shrub species differs from in northern areas, denoting their particular identity with the phytosociological name of AcaenoMulgaraetum tridentis. The lignotuber of Mulguraea tridens has a low rate of post-fire regrowth, so it is a community sensitive to fire. Therefore regeneration by seeds is crucial to its natural post-disturbance recovery. We suggest that this information may be useful for national park managers, allowing them to incorporate new biodiversity information into management programs, and develop strategies for post-fire ecological restoration for this scrub.

\section{Key words:}

Mulguraea tridens, fire, fitosociology, vegetation, Torres del Paine, ecological restoration.

\section{INTRODUCCIÓN}

El Parque Nacional Torres del Paine (PNTP) (Provincia de Última Esperanza, Región de Magallanes, Chile), se extiende entre $49^{\circ} 21^{\prime}$ y $51^{\circ} 08^{\prime}$ de latitud Sur y $73^{\circ} 07^{\prime}$ y $74^{\circ} 52^{\prime}$ de longitud Oeste, fue creado en 1959, y designado como Reserva de la Biósfera en 1978 (CONAF, 2007). En su sección nor-oeste el clima es frío y lluvioso por la influencia del cinturón de vientos del oeste (Moy et al. 2008), el Campo de Hielo Sur y la Cordillera de Paine que presentan varios glaciares (Garay \& Guineo, 2003), y en el sureste el relieve desciende presentando colinas de suaves pendientes hasta llegar a las planicies más secas de la estepa patagónica ubicada a sotavento de la Cordillera Paine (Pisano, 1974). La abrupta elevación del macizo montañoso, al que el Parque Nacional debe su nombre y domina en su posición central, genera por efecto de sombra de lluvia un drástico gradiente ambiental que promueve, en una franja de $50 \mathrm{~km}$, un descenso abrupto de las precipitaciones que varía desde unos 1.400 mm hasta unos $350 \mathrm{~mm}$ al año en su zona más árida (Pisano, 1974). Esta alta heterogeneidad ambiental permite el establecimiento de unidades de vegetación muy diversas, configurando un atractivo peculiar y representativo de la vegetación en la Ecorregión Magallánica Subantártica (sensu Rozzi et al. 2012).

En la vegetación del PNTP se pueden distinguir las siguientes provincias bióticas: estepa patagónica, matorral xerófito, matorral mesófito, bosque magallánico caducifolio y desierto andino (Pisano, 1974). Para Domínguez (2012) las macrounidades de vegetación que pueden distinguirse en el PNTP son: estepa patagónica, matorral preandino, bosque magallánico, turberas esfagnosas y pulvinadas y desierto andino. El matorral de Mata Negra (Mulgaraea tridens), se ubica en la Estepa patagónica que es coincidente en ambas clasificaciones. Esta formación esteparia, se localiza en el sector sur-oriental del PNTP, y presenta un relieve con llanuras de condición semidesértica muy secas con una precipitación promedio anual no superior a $350 \mathrm{~mm}$, muy ventoso, con alta evapotranspiración y carente de árboles y bosques (Pisano, 1974). Dominan en ella estepas de tipo herbáceo formadas por pastos perennes y duros (coirones) que crecen amacollados, entre los que dominan Festuca gracillima Hook. f. y Festuca magellanica Lam. La matriz duriherbosa que forman estos coirones es interrumpida por fragmentos dispersos del matorral de Mata Negra, con características xerofíticas (Vidal, 2007).

Los matorrales esteparios de Mata Negra han sido muy afectados por los últimos incendios acontecidos en el PNTP por acción de visitantes (Vidal, 2012). Por esta razón, es muy importante poder contar con una línea base que permita estudiar los cambios provocados por dichos incendios en la estructura de esta comunidad. Además, estos matorrales están dominados por 


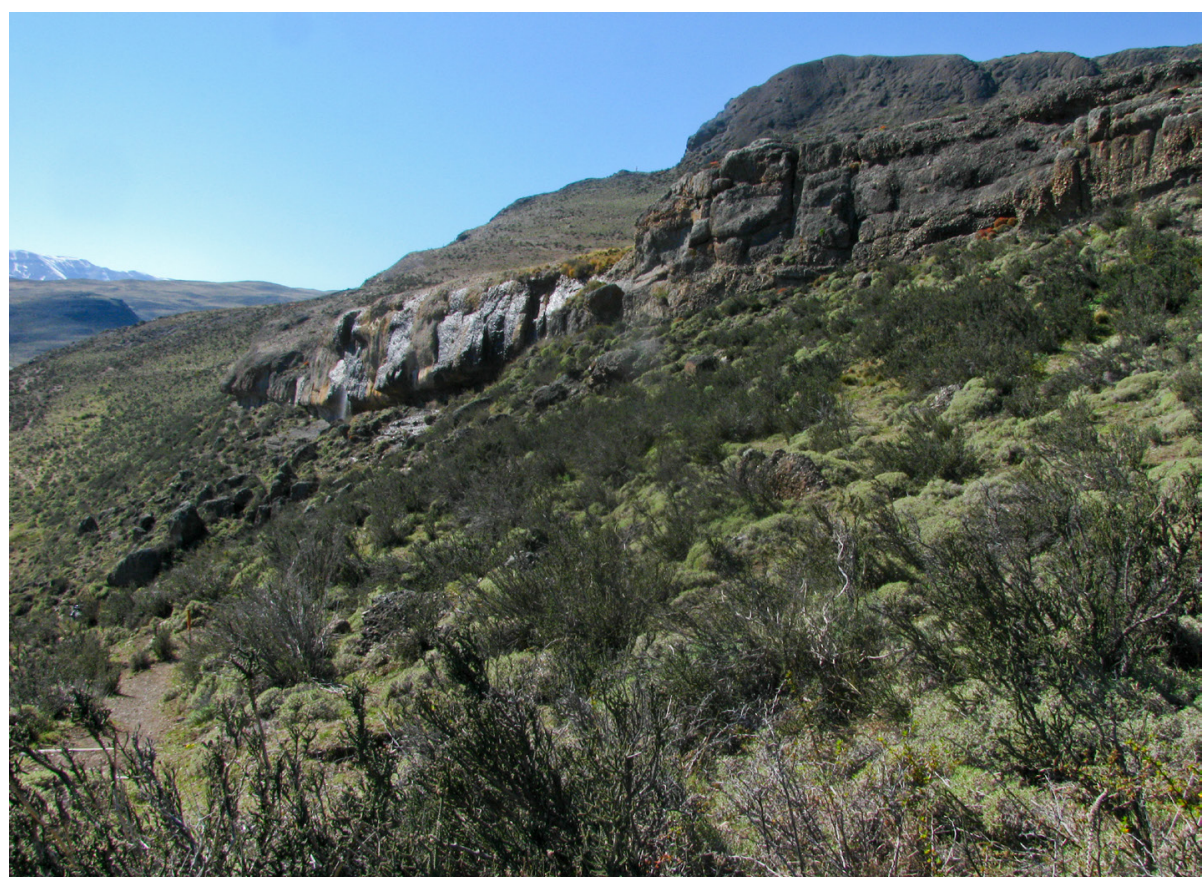

Fig. 1. Fisonomía del matorral de Mata Negra (Mulguraea tridens [Lag.] N. O'Leary \& P. Peralta) en el sector de Laguna Amarga en el Parque Nacional Torres del Paine.

especies con problemas de conservación (e.g. Mulguraea tridens, vulnerable) (Benoit, 1989) y "son únicos en su tipo dentro de un área silvestre protegida en Chile" (Domínguez et al. 2006). Esto se hace apremiante si se considera que el cambio climático y el turismo no regulado puede favorecer los incendios cada vez más frecuentes en ecosistemas naturales del PNTP. El presente estudio busca construir esa línea base utilizando la metodología fitosociológica, la cual permite describir y clasificar comunidades vegetales que, en último término, pueden asimilarse a los hábitats que habría que proteger o restaurar ecológicamente a través de actividades planificadas (Dierschke, 1994; Society for Ecological Restoration, 2004; Dengler, 2008). Consecuentemente, el objetivo de este trabajo consiste en describir el matorral de Mata Negra (Mulguraea tridens), con énfasis sobre sus atributos de composición florística y estructura comunitaria. Asimismo, entregamos antecedentes sobre la resiliencia al fuego de esta comunidad de interés para la conservación biológica (Benoit, 1989), que pueden ser de utilidad para su manejo con fines en restauración ecológica.

\section{MATERIALES Y MÉTODOS}

En fragmentos del matorral de Mata Negra existentes en varios sectores del Parque Nacional Torres del Paine se delimitaron y marcaron 35 parcelas de $25 \mathrm{~m}^{2}$ en las cuales se levantaron censos de vegetación (=relevés) con metodología fitosociológica de Europa Central (Braun-Blanquet, 1979; Jörg, 2003). Los censos cumplían los atributos de homogeneidad florística, fisonómica y ecológica sugeridos por Knapp (1984), constituídos aquí por la presencia dominante de la especie Mata Negra, configurando una fisionomía arbustiva muy característica (Fig. 1). Para cada censo de vegetación se hizo una lista completa de las especies vegetales presentes en la parcela y luego se determinó la abundancia de los individuos de cada una mediante el método no destructivo de estimar la cobertura en porcentaje (Ramírez et al. 1997). Para registrar coberturas bajo $1 \%$ se utilizaron los signos "+" (cruz) y "r" (erre) con su significado tradicional, el primero indicando varios individuos de la especie presentes y el segundo, sólo uno o dos de ellos (Mueller-Dombois \& Ellenberg, 1974), aunque para cálculos estadísticos estos signos se subieron 
a la unidad.

Cada parcela fue registrada con GPS utilizando coordenadas UTM y luego se determinó variables de sitio como su pendiente (en grados), su exposición y su altitud (en $\mathrm{m} \mathrm{snm}$ ). El relieve del fragmento donde se ubicaba la parcela fue clasificado como plano, cóncavo o convexo. Posteriormente se procedió a determinar la estratificación de la vegetación de cada parcela expresando en porcentaje la cobertura de cada estrato, que al sobreponerse pueden originar coberturas superiores al $100 \%$. Por último, para cada parcela se contabilizó el número total de especies vegetales presentes, diferenciando autóctonas de alóctonas.

Las especies de flora desconocidas fueron herborizadas y determinadas con posterioridad utilizando la literatura botánica pertinente (e.g. Moore, 1983; Henríquez et al. 1995; Vidal, 2007). $\mathrm{Su}$ nomenclatura y autorías fueron actualizadas en la página "The Plant List" del Jardín Botánico de Missouri, USA (Croft et al. 1999). El carácter autóctono o alóctono de las especies fue obtenido de Zuloaga et al. (2008). Las especies se clasificaron de acuerdo a las clases tradicionales, sin considerar los grupos establecidos recientemente que aunque son comprensibles para los botánicos, no son conocidos por otros científicos y profesionales (Wink, 2006).

Para caracterizar y clasificar el cormo vegetativo de las especies se utilizó el sistema de formas de vida establecido por Raunkiaer (1934) utilizando la clave entregada por Mueller-Dombois \& Ellenberg (1974). Raunkiaer distingue fanerófitos, caméfitos, hemicriptófitos, criptófitos y terófitos. Los primeros son plantas leñosas, tales como árboles, arbustos, agregándose especies de lianas, epífitas y parásitas. Los segundos corresponden a sub-arbustos y plantas pulviniformes (en cojín), los terceros son hierbas perennes, los cuartos hierbas con órganos subterráneos de reserva (bulbos, tubérculos o rizomas) y pueden separarse en geófitos (terrestres), acuáticos y palustres (de pantano). Los terófitos son hierbas anuales $y$ bianuales que tienen un ciclo de vida corto. La proporción de estas formas de vida corresponden al espectro biológico y entrega información sobre las condiciones del hábitat y su posición en la dinámica de degradación (Ramírez et al. 2012).
Características más específicas del cormo, tales como clonalidad, rebrote post-fuego y síndromes de dispersión, fueron tomadas de las definiciones sobre rasgos de plantas funcionales de Cornelissen et al. (2003), Ramírez et al. (2003), Paula y Labbé (2012), y Perez-Harguindeguy et al. (2013).

Los censos de vegetación se reunieron en una tabla para la cual se calculó la frecuencia (absoluta, porcentaje y relativa), cobertura (total, promedio y relativa) y sumando frecuencia y cobertura relativas se obtuvo un valor de importancia de acuerdo a Wikum \& Shanholtzer (1978) que sirve para ordenar las especies de la tabla y presenta un valor máximo posible de 200. Este valor de importancia carece de variación ya que sólo representa un número de orden. La asociación vegetal se nombró de acuerdo a las normas de nomenclatura fitosociológica vigentes (Izco \& del Arco, 2003).

Finalmente, se construyó una matriz de especies (en columnas) x unidades de muestreo (en filas), en donde se expresó las coberturas en porcentajes. También se construyó una segunda matriz de variables ambientales $\mathrm{x}$ unidades de muestreo. Las variables ambientales fueron: a) Tipo de relieve (plano, concavo, convexo); b) Pendiente (baja [0-25\%], moderada [26-60\%] y alta [61-100\%]); c) Aspecto; d) Cobertura de rocas (\%); e) Suelo desnudo (\%); f) Cobertura total (\%); g) Cobertura de especies nativas y; h) Cobertura de especies exóticas. Esta matriz fue sometida a un procedimiento de ordenación de escalamiento multidimensional no métrico (Legendre \& Legendre, 1998; Mc Cune \& Grace, 2002), con los resultados de la ordenación ajustados a las variables ambientales para evaluar gradientes de dispersión sitio-especies dentro del matorral de Mata Negra. Solo las variables ambientales asociadas a las coordenadas estadísticamente significativas fueron incluidas. Se aceptó un nivel de significancia de $p<0,10$ debido a la naturaleza exploratoria del análisis.

\section{RESULTADOS}

Los resultados analizados a continuación tuvieron como base la tabla inicial conformada por 68 especies (Anexo 1) y 35 censos. En primer lugar se tratarán los atributos estructurales de las parcelas muestreadas, luego los atributos de composición 
y estructura comunitaria que incluye la flora y el análisis de la tabla de vegetación.

\section{Atributos estructurales de las parcelas}

La Tabla 1 muestra las coordenadas geográficas UTM para la ubicación de los 35 censos levantados en el matorral estepario de Mulguraea tridens en el Parque Nacional Torres del Paine. Todas las parcelas presentaron pendiente suave la que osciló entre $15^{\circ}$ como máximo y $2^{\circ}$ como mínimo, con un promedio de $7,8^{\circ}$. El macro relieve del lugar trabajado se consideró como plano en 13 parcelas, cóncavo en 12 y convexo en 10 de ellas. Las exposiciones Norte (también Noroeste y Noreste) fueron las más frecuentes con 26 censos, las exposiciones Sur sólo se presentaron en 4 parcelas, Este 4 y Oeste 1, lo que indica que este matorral xérico prefiere la exposición más cálida. La altitud de las parcelas presentó como promedio $247 \mathrm{~m}$ snm con un máximo de $310 \mathrm{~m}$ snm y un mínimo de $160 \mathrm{~m}$ snm.

\section{Atributos de composición y estructura comunitaria}

La cobertura promedio del estrato arbustivo de todas las parcelas alcanzó a $48,4 \%$ con un máximo de $71 \%$ y un mínimo de $25 \%$, siendo éste el estrato con mayor cobertura. Le siguen los estratos sub-arbustivo y herbáceo, que en conjunto formaron el estrato inferior, con un promedio de $26,2 \%$ de cobertura (máximo de $46 \%$ y un mínimo de $10 \%)$. De acuerdo a lo anterior, la cobertura vegetal promedio de las parcelas fue de $74,6 \%$ y por lo tanto, un promedio de $25,43 \%$ de la superficie de ella corresponde a suelo desnudo, incluidas rocas.

El anexo 1 contiene una breve caracterización de las 68 especies vegetales encontradas en las 35 parcelas analizadas, en ella se entrega nombre científico y autor, el grupo sistemático al que pertenecen, la familia, nombres comunes, origen y forma de vida, dispersión y capacidad de rebrote. De las 68 especies que forman la flora, 52 (76,47\%) son Dicotiledóneas y 16 (23,53\%) Monocotiledóneas. Al considerar la cobertura, las Dicotiledóneas suben a 91,24\%, mientras que las Monocotiledóneas bajan a $8,76 \%$ presentándose con escasa abundancia.

La mayoría de las especies vegetales presentes corresponde a especies autóctonas, con 40 especies en total (promedio 12,3 $\pm 2,83 \mathrm{SD}$ ) y 28 de ellas alóctonas (promedio 4,5 $\pm 2,3 \mathrm{SD}$ ) (Fig. 2, Anexo 1). Sin embargo al considerar la cobertura, las autóctonas representan en promedio un $65,2 \%$ ( $\pm 12,5 \mathrm{SD}$ ) de la abundancia en cobertura, mientras que las alóctonas representan en promedio sólo un 9,1\% ( $\pm 10,2$ SD) de la abundancia (Fig. 2). La dominancia de especies autóctonas caracteriza la comunidad vegetal como una asociación primaria, original del lugar, aunque la presencia de especies alóctonas demuestra influencia antrópica sobre ella, pero podría deberse también a la disponibilidad de espacio, dado que un porcentaje importante del suelo de cada parcela

\section{a) Riqueza de especies}

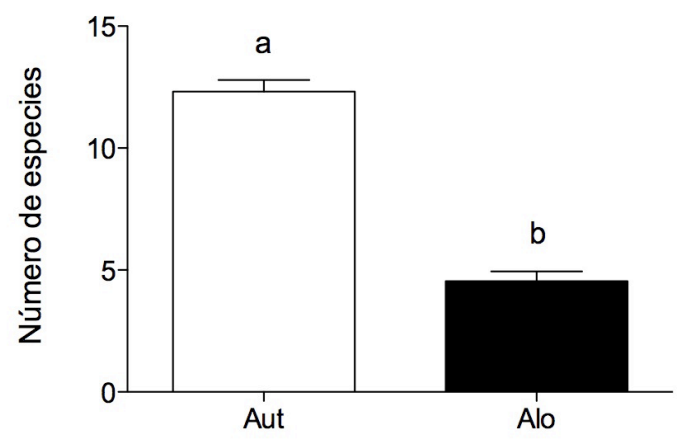

b) Abundancia

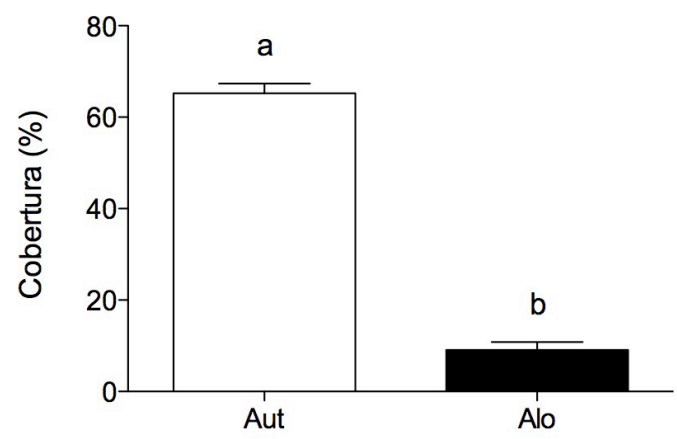

Fig. 2. Riqueza (a) y abundancia (b) de especies de plantas vasculares registradas para el matorral de Mata Negra (Mulguraea tridens [Lag.] N. O'Leary \& P. Peralta) en el Parque Nacional Torres del Paine ( $t$-test, $p<0,05)$. Las barras muestran promedios y error estándar. Aut= especies autóctonas; Alo= Especies alóctonas. 
Tabla 1. Atributos espaciales y de micrositio para los censos del matorral de Mata Negra registrados en en Parque Nacional Torres del Paine.

\begin{tabular}{|c|c|c|c|c|c|c|}
\hline $\mathrm{N}^{\circ}$ & $\begin{array}{l}\text { UTM } \\
\text { (me) }\end{array}$ & $\begin{array}{l}\text { UTM } \\
\text { (ms) }\end{array}$ & $\begin{array}{c}\text { Inclinación } \\
\left({ }^{\circ}\right)\end{array}$ & $\begin{array}{c}\text { Exposición } \\
\text { Aspecto }\end{array}$ & $\begin{array}{c}\text { Relieve } \\
\text { Tipo }\end{array}$ & $\begin{array}{c}\text { Altitud } \\
\text { (m) }\end{array}$ \\
\hline 01 & 654270 & 4349587 & 10 & Oeste & cóncavo & 174 \\
\hline 02 & 654139 & 4349604 & 5 & Norte & plano & 160 \\
\hline 03 & 654552 & 4349736 & 10 & Norte & cóncavo & 181 \\
\hline 04 & 654612 & 4349551 & 3 & Norte & plano & 201 \\
\hline 05 & 654136 & 4349468 & 7 & Norte & cóncavo & 184 \\
\hline 06 & 654232 & 4349466 & 12 & Norte & cóncavo & 188 \\
\hline 07 & 654307 & 4349312 & 4 & Noreste & plano & 213 \\
\hline 08 & 653915 & 4349245 & 15 & Norte & cóncavo & 272 \\
\hline 09 & 654156 & 4349166 & 9 & Norte & cóncavo & 234 \\
\hline 10 & 654047 & 4349215 & 5 & Noroeste & plano & 243 \\
\hline 11 & 653939 & 4349113 & 5 & Noreste & convexo & 282 \\
\hline 12 & 654054 & 4349114 & 13 & Noreste & cóncavo & 256 \\
\hline 13 & 654022 & 4349050 & 15 & Noreste & cóncavo & 274 \\
\hline 14 & 653914 & 4348994 & 5 & Noreste & cóncavo & 301 \\
\hline 15 & 654270 & 4349059 & 12 & Noroeste & convexo & 258 \\
\hline 16 & 654342 & 4349198 & 5 & Noreste & cóncavo & 226 \\
\hline 17 & 654596 & 4349268 & 5 & Sureste & cóncavo & 222 \\
\hline 18 & 654616 & 4349180 & 10 & Noreste & plano & 231 \\
\hline 19 & 654662 & 4349076 & 2 & Norte & plano & 245 \\
\hline 20 & 654502 & 4349068 & 5 & Norte & plano & 249 \\
\hline 21 & 654654 & 4348949 & 10 & Este & convexo & 260 \\
\hline 22 & 654709 & 4348850 & 2 & Norte & plano & 263 \\
\hline 23 & 654485 & 4348590 & 4 & Noreste & plano & 287 \\
\hline 24 & 654657 & 4348427 & 5 & Norte & plano & 294 \\
\hline 25 & 654719 & 4348529 & 4 & Norte & plano & 286 \\
\hline 26 & 654369 & 4358517 & 10 & Noroeste & plano & 290 \\
\hline 27 & 654458 & 4348002 & 10 & Este & convexo & 297 \\
\hline 28 & 654272 & 4347610 & 5 & Sureste & convexo & 174 \\
\hline 29 & 654205 & 4347242 & 10 & Este & convexo & 271 \\
\hline 30 & 654501 & 4347214 & 12 & Suroeste & convexo & 253 \\
\hline 31 & 654545 & 4346626 & 4 & Noreste & cóncavo & 251 \\
\hline 32 & 654413 & 4346467 & 10 & Este & plano & 272 \\
\hline 33 & 654448 & 4346363 & 5 & Sureste & convexo & 268 \\
\hline 34 & 654186 & 4346611 & 10 & Norte & convexo & 280 \\
\hline 35 & 653958 & 4346520 & 15 & Noreste & convexo & 310 \\
\hline
\end{tabular}


permanece desnudo y que las malezas actuando como oportunistas lo colonizarían.

En el espectro biológico dominan los hemicriptófitos, es decir, hierbas perennes, con 39 especies en total. De estas los hemicriptófitos autóctonos promediaron 5,8 ( \pm 2,1 SD) especies por censo de vegetación, mientras que los hemicriptófitos alóctonos promediaron 2,4 $( \pm 1,3$ SD) especies (Fig. 3). Los terófitos, en tanto, estuvieron constituidos por 15 especies, de entre las cuales los terófitos autóctonos promediaron $1,0$ ( $\pm 0,7 \mathrm{SD})$ especies, mientras que los terófitos alóctonos los superaron levemente en riqueza de especies (1,6 \pm 1,4 SD). Los caméfitos, subarbustos pulvinados principalmente, alcanzan a 7 especies, mientras que los geófitos (hierbas con órganos subterráneos de reserva) sólo presentaron 4 especies. Los fanerófitos, es decir, plantas leñosas arbustivas alcanzaron presentaron la menor cantidad de especies (sólo 3 especies). Este espectro biológico caracteriza a un clima estepario extremo con mucha sequía reflejada en el alto número de plantas anuales. Al considerar la cobertura de las mismas, las proporciones cambian drásticamente, ya que ahora los fanerófitos arbustivos representaron el 56,8\% de la cobertura total (promedio 41,6 \pm 13,1 SD) (Fig. 3 ), dominando ampliamente por sobre las otras formas de vida (Fig. 3). Los geófitos presentaron sólo un $1,27 \%$ de la cobertura, lo que se justifica por lo efímero y reducido de su cormo aéreo. El espectro biológico que considera la abundancia (cobertura) de los individuos de una especie, refleja

a) Raunkiaer - Riqueza de Especies

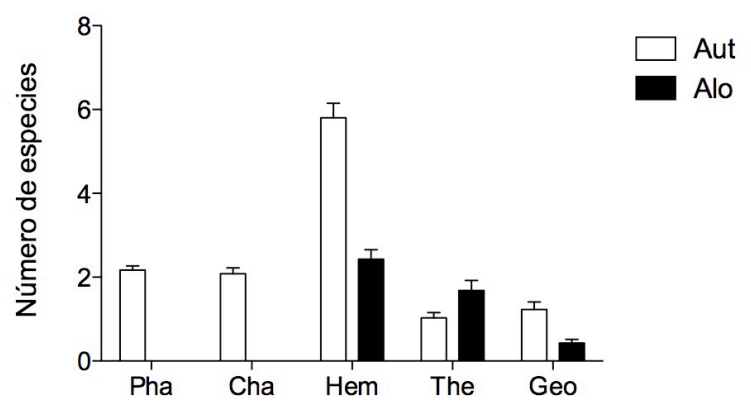

mucho mejor la realidad del fitoclima y pone de manifiesto la importancia de los arbustos, dándole un carácter estepario xérico.

Los síndromes de dispersión presentan una mayor variación contabilizándose 12 especies anemócoras, 10 epizoócoras, 3 endozoócoras, 3 autócoras y especies con dispersión no conocida, que seguramente incluyen barocoría, autocoría y policoría que son síndromes muy frecuentes. Lo anterior destaca la importancia del viento y los animales en la dispersión de propágulos en la estepa patagónica, de la que aún falta mucho por conocer (Anexo 1). Clonalidad mediante reproducción vegetativa sólo se constató en 26 de las 68 especies, 10 de ellas con órganos persistentes bajo el sustrato y 16 sobre el suelo. En consecuencia, existen 42 especies que no presentan reproducción vegetativa. Estos resultados concuerdan con el hecho de que sólo 31 especies pueden brotar vegetativamente después de la acción del fuego.

El anexo 1 corresponde al listado de especies de plantas vasculares y a sus principales atributos de historia de vida (e.g. formas de vida, dispersión) y comunitarios (e.g. valor de importancia, frecuencia) para la asociación que denominamos Acaeno-Mulgaraetum tridentis asoc. nova por las especies nativas diferenciales Acaena pinnatifida y Mulgaraea tridens, que corresponde a un matorral estepario al parecer primario, es decir originario en el Parque Nacional Torres del Paine (Provincia de Última Esperanza, Región de Magallanes, Chile). En

\section{b) Raunkiaer - Abundancia de Especies}

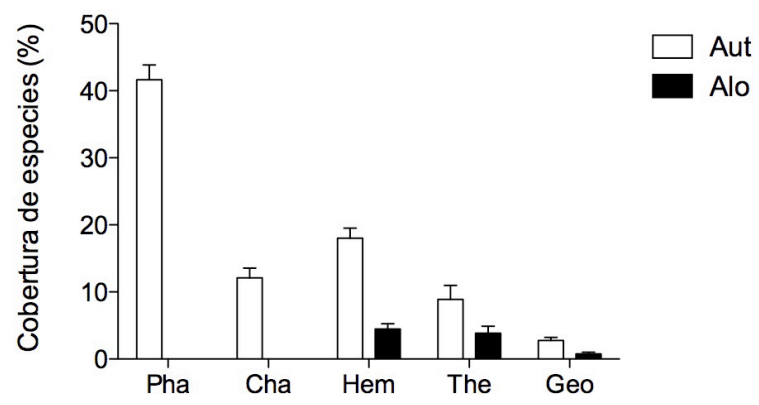

Fig. 3. Riqueza (a) y abundancia (b) de especies autóctonas (Aut) y Alóctonas (Alo) en el matorral de Mata Negra (Mulguraea tridens [Lag.] N. O'Leary \& P. Peralta) en Torres del Paine, de acuerdo a las formas de vida de Raunkiaer (Pha= fanerófitos; Cha= Caméfitos; Hem= Hemicriptófitos; The = Terófitos; Geo= Geófitos). Las barras muestran valores promedio y error estándar. 
dicho anexo figuran los valores de importancia y en ella dominó ampliamente Mulgaraea tridens con 54,9, valor que corresponde aproximadamente a una cuarta parte de todas las especies que en conjunto alcanzaron a 200 de valor de importancia. Mucho más abajo le siguieron Mulinum spinosum con 16,9, Senecio patagonicus Hook. \& Arn. con 8,0, Berberis microphylla con 6, 4 y Baccharis magellanica con 6,3 de valor de importancia. La especie Acaena pinnatifida escogida como diferencial, apareció en el lugar 9 con un valor de importancia de 4,2.

En relación a las frecuencia de las especies, Mulgaraea tridens es la única especie que apareció en los 35 censos con un $100 \%$. Le siguieron en orden decreciente, Mulinum spinosum que se presentó en 33 censos y Senecio patagonicus en 22, coincidiendo con el valor de importancia presentado por cada una. A continuación se encontró Adesmia lotoides presente en 21 censos y Acaena pinnatifida y Microsteris gracilis (Hook.) Greene presentes en 20 censos cada uno. Sin embargo, al considerar la cobertura se mantuvo casi el mismo orden que en el valor de importancia, aunque Baccharis magellanica subió al cuarto lugar y Berberis microphylla, descendió al quinto.

$\mathrm{Al}$ ordenar el porcentaje de frecuencia de todas las especies presentes en la comunidad se puede observar una alta homogeneidad de la comunidad, lo que se confirma con el hecho de que las 5 primeras especies reunieron sobre el $70 \%$ de la cobertura total de la asociación (Tabla 2).

Tabla 2. Número de especies de plantas vasculares cada clase de frecuencia presentes en el matorral de Mata Negra Acaeno-Mulguraetum tridentis en el Parque Nacional Torres del Paine.

\begin{tabular}{cccc}
\hline Clase & Rangos & Especies & Porcentaje \\
A & $80-100$ & 2 & 2,94 \\
B & $60-79$ & 2 & 2,94 \\
C & $40-59$ & 16 & 23,53 \\
D & $20-39$ & 15 & 22,06 \\
E & $0-19$ & 33 & 48,53 \\
\hline Total & & 68 & 100,00 \\
\hline
\end{tabular}

Tabla 3. Presencia de especies diferenciales en matorrales del Berberido-Mulguraetum (ex-Junielletum) tridentis (en Argentina) y en matorrales del Acaeno-Mulgaraetum tridentis en el Parque Nacional Torres del Paine.

\begin{tabular}{|c|c|c|}
\hline Especie & Argentina & Torres del Paine \\
\hline Mulguraea tridens & $\mathrm{x}$ & $\mathrm{x}$ \\
\hline Berberis heterophylla & $\mathrm{x}$ & . \\
\hline Nassauvia glomerulosa & $\mathrm{x}$ & . \\
\hline $\begin{array}{l}\text { Nardophyllum } \\
\text { obtusifolium }\end{array}$ & $\mathrm{x}$ & . \\
\hline Chuquiraga avellanedae & $\mathrm{x}$ & . \\
\hline Poa ibanii & $\mathrm{x}$ & . \\
\hline Stipa ibari & $\mathrm{x}$ & . \\
\hline Burkartia lanigera & $\mathrm{x}$ & . \\
\hline Berberis microphylla & $\mathrm{x}$ & $\mathrm{x}$ \\
\hline Nassauvia abbreviata & $\mathrm{x}$ & $\mathrm{x}$ \\
\hline Clinopodium darwinii & $\mathrm{x}$ & . \\
\hline Olsinium biflora & $\mathrm{x}$ & $\mathrm{x}$ \\
\hline Huanaca acaulis & $\mathrm{x}$ & . \\
\hline Azorella trifurcata & $\mathrm{x}$ & . \\
\hline Ephedra frustillata & $\mathrm{x}$ & . \\
\hline Polygala darwiniana & $\mathrm{x}$ & $\mathrm{x}$ \\
\hline Total & 16 & 5 \\
\hline
\end{tabular}

Descripción de la asociación vegetal

Matorral de mata negra

Matorral de Mulgaraea tridens

Acaeno-Mulgaraetum tridentis asoc. nova Asociación de Acaena pinnatifida Ruiz \& Pav. y Mulgaraea tridens (Lag.) N. O'Leary \& P. Peralta

Se trata de un matorral primario nativo de la estepa patagónica chilena muy bien representado en el Parque Nacional Torres del Paine. Normalmente, aparece en condiciones muy secas que no superan los $500 \mathrm{~mm}$ anuales promedio de precipitación (Pisano, 1974), siendo afectado además, por los frecuentes vientos propios de la estepa que provocan un aumento de la evapo-transpiración 
NMDS - SITIOS

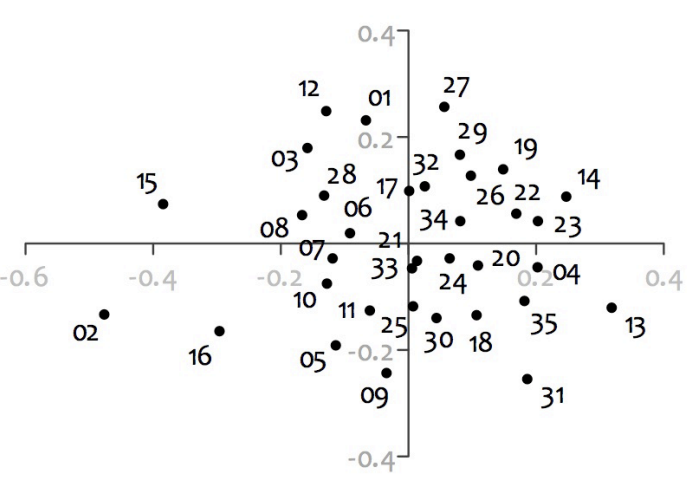

NMDS - ESPECIES

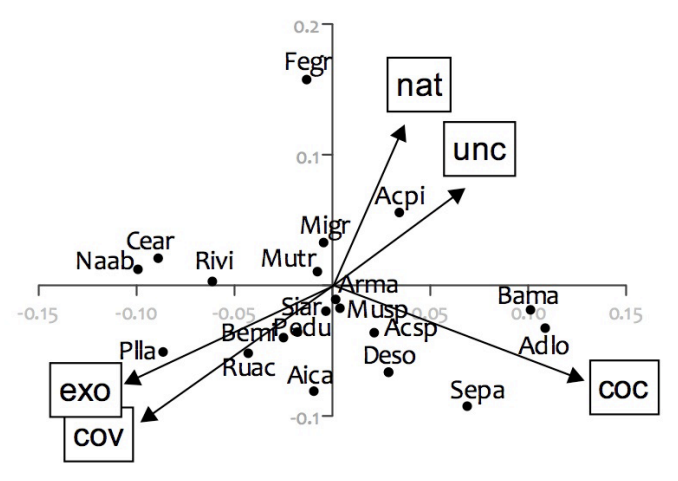

Fig. 4. Analisis de ordenación según el modelo de escalamiento multidimensional no métrico (NMDS) (Stress=0,26), mostrando la posición de las 35 unidades de muestreo (izquierda) y las especies junto a variables ambientales que mejor se ajustan a las dos primeras dimensiones (derecha) $(p<0,10)$. Acrónimos para las variables ambientales: $\operatorname{coc}=$ relieve cóncavo; $\mathrm{cov}=$ relieve convexo; exo= cobertura de especies exóticas; nat= cobertura de especies nativas; unc= covertura del dosel.

de las plantas, dándole a la comunidad sus características de matorral xerofítico. Es un matorral triestratificado con un dosel superior muy denso, homogéneo, ramoso e intrincado de Mulgaraea tridens, un estrato arbustivo inferior de Mulinum spinosum y un estrato basal inferior formado por hierbas que aprovechan el espacio disponible, dejado por las dominantes. En el estrato herbáceo abundan hierbas anuales (e.g. Aira caryophyllea), y otras especies alóctonas abundantes (e.g. Rumex acetosella), que crecen con condiciones de sequía edáfica con relieve convexo (Fig. 4). Sobre el dosel del matorral suelen sobresalir individuos aislados de Berberis microphylla, Senecio patagonicus y Bacharis magellanica en sectores de relieve cóncavo, donde se acumula mayor humedad edáfica (Fig. 4). La abundancia de Mulgaraea tridens le da un color verde oscuro a negruzco a este matorral, lo que destaca en la matriz esteparia graminosa de Festuca gracillima, de color más amarillento que los rodea. Esta última especie caracteriza los sitios menos invadidos en sectores con dominancia de especies nativas (Fig. 4). También destaca la floración de Mulgaraea tridens que lo cubre con flora blancas a veces, rosadas o violáceas.

\section{DISCUSIÓN}

Los matorrales de mata negra del Parque Nacional Torres del Paine habían sido estudiados en Chile por Pisano (1974) desde el punto de vis- ta fitogeográfico, con el nombre de Verbenetum tridens, dándoles una ubicación en la estepa patagónica. Sin embargo, esta primera descripción fitogeográfica de esta estepa arbustiva no se asocia una tabla de vegetación que permita validarla como tal desde el punto de vista fitosociológico, por lo cual pasa a la sinonimia (Izco \& Del Arco, 2003). Posteriormente, es mencionada por Vidal (2007) como un matorral xerofítico también para las Torres del Paine. Por último, Mansilla (2007) la estudia como una comunidad de Junellia tridens (Lag.) Moldenke (Mulgarea tridens) objeto de incendios antropogénicos, aceptando para ella el nombre propuesto por Roig et al. (1985) de Berberido-Verbenetum tridentis, asociación que fue ratificada más tarde por Roig (1994) pero de acuerdo al presente estudio, dicha asociación vegetal descrita para el Norte de la Transecta Botánica de la Patagonia Austral en territorio argentino, es diferente a la asociación que se presenta en Torres del Paine y en el Parque Nacional Pali Aike en la Región de Magallanes. La Tabla 2 compara especies de la asociación Berberido-Verbenetum tridentis que Roig (1994) presenta como diferenciales de las variantes húmeda y seca de la misma comunidad. Se observa que de 16 especies mencionadas por dicho autor sólo 5 , un tercio, es común con la asociación estudiada en Torres del Paine, todas ellas corresponden a la variante húmeda del matorral de mata negra. Lo anterior confirma lo que se propone en este trabajo que la comunidad 
de mata negra de Torres del Paine se trata de una nueva asociación vegetal para la cual se propone el nombre de Acaeno-Mulgaraetum tridentis asoc. nova que calza muy bien en la clasificación sintaxonómica propuesta por Roig et al. (1985) como se indica a continuación.

Clase: Festucetea gracillimae Roig et al. 1985 Orden: Nardophyllo-Festucetalia Roig. et al. 1985 Alianza: Stipo-Nasauvio ulicinae Roig et al. 1985 Asociación: Berberido-Verbenetum tridentis Roig et al. 1985 (en Argentina) Asociación: Acaeno-Mulgaraetum tridentis asoc. nova (en Chile)

Para Roig et al. (1985) y Roig (1994) Mulgaraea tridens es una especie que puede ocupar rápidamente áreas degradadas por el pastoreo o denudadas por el fuego, este último favorecería la instalación de ella y su comunidad, basando sus observaciones en una parcela de $25 \mathrm{~m}^{2}$ en una matorral de mata negra recientemente quemado y en el cual contabiliza 24 individuos de Mulgarea tridens muertos, uno rebrotado a partir de tocón y 102 plántulas de la misma especie. Esta observación fue realizada en territorio argentino y se contradice con aquellas realizadas por Mansilla (2007) en el Parque Nacional Torres del Paine quien realizó un conteo sobre 1.149 individuos de Mulguraea tridens en sitios afectados dos años después de un incendio (incendio 2005). En este estudio, Mansilla (2007) mostró que apenas 28 individuos $(2,4 \%)$ se habían regenerado sexualmente a partir de semillas, mientras que 127 individuos (11,0\%) se habían regenerado vegetativamente a partir del lignotúber quemado. El 86,5\% de los individuos registrados, sin embargo, no presentaba regeneración, dando cuenta de que la especie es sensible a los efectos del fuego.

Las mediciones de Mansilla (2007) realizadas en parcelas no incendiadas y quemadas del Acaeno-Mulgaraetum tridentis no muestran grandes diferencias en el número de especies de esas dos situaciones, ya que en el primer caso hay 43 especies y en el segundo, 48 presentando aquellas nuevas del área quemadas, bajo porcentaje de cobertura. Así en las áreas quemadas aparecen 12 nuevas especies de las cuales sólo tres, Adesmia boronoides Hook. f., Draba verna L. y Leu- canthemum vulgare Lam. no se encontraron en las parcelas observadas en este trabajo. En las parcelas quemadas desaparecieron 7 especies, Vicia magellanica Hook. f., Senecio patagonicus, Phacelia braticantha, Gamochaeta spiciformis (Sch. Bip.) Cabrera, Colobanthus subulatus (d'Urv.) Hook. f., Dactilys glomerata L. y Bolax gummifera (Lam.) Spreng., de estas solo 3 tres no aparecen en este trabajo: P. braticantha, G. spiciformis y B. gummifera. Las diferencias más grandes encontradas por Mansilla (2007) se presentan en los porcentajes de cobertura de las especies presentes en áreas quemadas y no quemadas, ya que Acaena pinnatifida, Agrostis capillaris L., Cerastium arvense, Plantago lanceolata L., Poa annua L., Poa pratensis L., Rumex acetosella, Taraxacum officinale F. H. Wigg. y Viola maculata Cav. aumentan su cobertura en aquellas áreas quemadas, 7 de ellas son alóctonas oportunistas que aprovechan el espacio disponible para expandirse (Labbé, 2013). El caso más interesante lo presenta Rumex acetosella (romacilla) especie indicadora de quema (Ramírez et al. 1991) y cuyo aumento de cobertura como consecuencia de incendios había sido ya observado por Dollenz (1991) y Dollenz \& Ivanovic (1996). Una disminución de la cobertura en áreas quemadas fue presentada por Festuca gracillima, Mulgaraea tridens y Mulinum spinosum, especies nativas importantes en la flora del AcaenoMulgaraetum tridentis. De estos datos se puede concluir que el fuego favorece el desarrollo de las alóctonas e inhibe considerablemente el desarrollo de las especies autóctonas.

En el presente trabajo se registraron 68 especies, pero si a ellas se suman las contempladas por Mansilla (2007), la riqueza de especies aumenta a 80, dando cuenta de una comunidad muy rica en especies. El porcentaje de especies alóctonas aumentó de $28 \%$ a $31 \%$ en las áreas quemadas (Mansilla, 2007). En el presente trabajo, sin embargo, el porcentaje de especies alóctonas sube a $41 \%$, lo cual puede indicar que las parcelas medidas habían sido ya sometidas al fuego con mucha anticipación, aunque también esto puede deberse a la influencia del ganado doméstico (Caicheo, 2006), a la herbivoría por guanaco, o al aumento de visitantes del parque, ya que de acuerdo a Vidal (2005) y Vidal (2012), los turistas también son vectores de malezas. El aumento de alóctinas 
comentado no deja de ser preocupante y debería de alguna manera controlarse ya que estas plantas alóctonas alteran la estructura y el funcionamiento del matorral de Mulgaraea tridens.

Finalmente, el espectro biológico de la flora del matorral de Mulgaraea tridens indica que fanerófitos arbustivos y caméfitos leñosos, contribuyen con combustible para los incendios, los que pueden ser fácilmente iniciados por la presencia de terófitos y hemicriptófitos herbáceos alóctonos que siempre presentan necromasa aérea seca, siendo esto un excelente comburente (Ramírez et al. 1989, Navarro-Cerrillo et al. 2008). Incluso, dicha necromasa seca podría provocar igniciones espontáneas por causas naturales. Debido a estos atributos, el matorral de Mata Negra es una unidad vegetacional altamente amenazada por incendios en Torres del Paine, los que han dañado seriamente la biodiversidad y heterogeneidad paisajística en el área mencionada (Vidal, 2015).

\section{BIBLIOGRAFÍA}

Benoit, I. (1989). Red book on Chilean terrestrial flora. Santiago: Corporación Nacional Forestal.

Braun-Blanquet, J. (1979). Fitosociología, bases para el estudio de las comunidades vegetales. Madrid, España: H. Blume Ediciones.

Caicheo, A. (2006). Ensayos de germinación en especies arbustivas nativas con valores ornamental de la XII Región. Tesis de grado. Punta Arenas, Chile. Universidad de Magallanes.

Cornelissen, J. H. C., Lavorel, S., Garnier, E., Díaz, S., Buchmann, N., Gurvich, D. E., Reich, P. B.,...Poorter, H. (2003). A handbook of protocols for standardized and easy measurement of plant functional traits worldwide. Australian Journal of Botany, 51, 335-380.

Corporación Nacional Forestal (CONAF) (2007). Plan de Manejo Parque Nacional Torres del Paine. Punta Arenas.

Croft, J., Cross, N., Hichcliffe, S., Lughanda, E. N., Stevens, P. F., West, J. G., \& Whitbread, G. (1999). Plant names for the 21st century: the International Plant Name
Index, a distributed data source of general accessibility. Taxon, 48, 317-324.

Dengler, J., Chytry, M., \& Ewald, J. (2008). Phytosociology. In S. E. Jøgensen \& B. D. Fath (Eds.), Encyclopedia of Ecology (pp. 2767-2779). Oxford, England: Elsevier.

Dierschcke, H. (1994). Pflanzensoziologie Grundlagen und Methoden. Alemania, Stuttgart: Eugen Ulmer

Dollenz, O. (1991). Capacidad de colonización de Rumex acetosella L. (Vinagrillo) en comunidades perturbadas. Anales del Instituto de la Patagonia, 20, 61-66.

Dollenz, O., \& Ivanovic, J. (1996). Sucesión secundaria en un pastizal incendiado en el Parque Nacional Torres del Paine", Magallanes, Chile. Anales del Instituto de la Patagonia, 24, 15-28.

Domínguez, E. (2012). Flora nativa Torres del Paine. Santiago, Chile: Ocho Libros Editores.

Domínguez, E., Elvebakk, A., Marticorena, C., \& Pauchard, A. (2006). Plantas introducidas en el Parque Nacional Torres del Paine, Chile. Gayana Botánica, 63, 131-141.

Garay, G., \& Guineo, O. (2003). Fauna, flora y montaña de Torres del Paine. Amigos de la Vida Silvestre, Punta Arenas, Chile.

Henríquez, J. M., Pisano, E., \& Marticorena, C. (1995). Catálogo de la flora vascular de Magallanes (XII Región), Chile. Anales del Instituto de la Patagonia, 23, 5-30.

Izco, J., \& Del Arco, M. (2003). Código Internacional de Nomenclatura Fitosociológica. Materiales Didácticos Universitarios, Serie Botánica, 2, 1-154.

Jörg, E. (2003). A critique for phytosociology. Journal of Vegetation Science, 14, 291296.

Knapp, R. (1984). Considerations on quantitative parameters and qualitative attibutes in vegetation analysis and in phytosociological relevés. In Knapp, R. (Ed.), Sampling methods and taxon analysis in vegetation science (pp.77-119). La Haya: Dr. W, Junk Publishers.

Labbé, D. L. (2013). Efecto del fuego sobre la viabilidad y germinación de semillas en ecosistemas Patagónicos. Tesis Ingeniería 
en Conservación de Recursos Naturales. Valdivia, Chile: Facultad de Ciencias Forestales y Recursos Naturales, Universidad Austral de Chile.

Legendre, P., \& Legendre, L. (1998). Numerical ecology. Amsterdam: Elsevier.

Mansilla, H. (2007). La comunidad de Junellia tridens y los efectos del fuego en el Parque Nacional Torres del Paine, Patagonia, Chile. Trabajo de grado. Punta Arenas, Chile: Facultad de Ciencias, Universidad de Magallanes.

Mc Cune, B., \& Grace, J. B. (2002). Analysis of ecological communities. Gleneden Beach, Oregon, USA: MJM Software.

Moore, D. (1983). Flora of Tierra del Fuego. Anthony Nelson, Shropshire \& Missouri Botanical Garden.

Moy, C. M., Dunbar, R. B, Moreno, P. I., Francois, J.P., Villa-Martínez, R., Mucciarone, D. M., Guilderson, T. P., \& Garreaud, R. D. (2008). Isotopic evidence for hydrologic change related to the westerlies in SW Patagonia, Chile, during the last millenium. Quaternary Science Reviews, 27, 1335-1349.

Mueller-Dombois, D., \& Ellenberg, H. (1974). Aims and methods of vegetation ecology. New York, USA: Wiley \& Sons.

Navarro-Cerrillo, R., Hayas, A., García-Ferrer, A., Hernández, R., Duhalde, D., \& González, L. (2008). Caracterización de la situación posincendio en el área afectada por el incendio de 2005 en el Parque Nacional Torres del Paine (Chile) a partir de imágenes ultiespectrales. Revista Chilena de Historia Natural, 81, 95-110.

Paula, S., \& Labbé, D. L. (2012). Evaluación de los impactos del incendio Olguín (PN Torres del Paine) en la vegetación. Valdivia, Chile: Instituto de Ciencias Ambientales \& Evolutivas, Facultad de Ciencias, Universidad Austral de Chile.

Perez-Harguindeguy, N., Díaz, S., Garnier, E., Lavorel, S., Poorter, H., Jaureguiberry, P., Bret-Harte, M. S.,...Cornelissen, J. H. C. (2013). New handbook for standarised measurement of plant functional traits worldwide. Australian Journal of Botany, 61, 167-234.
Pisano, E. (1974). Estudio ecológico de la región continental sur del área andino-patagónica. II. Contribución a la fitogeografía de la zona del Parque Nacional Torres del Paine. Anales del Instituto de la Patagonia, 5, 59-104.

Ramírez, C., San Martín, C., \& Sempe, J. (1989). Cambios estacionales de tamaño de plantas, biomasa y fenología en una pradera antropogénica del Centro-Sur de Chile. Agro Sur, 17, 19-28.

Ramírez, C., Finot, V., San Martín, C. \& Ellies, A. (1991). El valor indicador ecológico de las malezas del Centro-Sur de Chile. Agro Sur, 19, 94-116.

Ramírez, C., San Martín, C., \& Ojeda, P. (1997). Muestreo y tabulación fitosociológica aplicados al estudio de los bosques nativos. Bosque, 18, 19-27.

Ramírez, C., Álvarez, M., \& San Martín, C. (2003). Diásporas y mecanismos de dispersión en praderas antropogénicas de la X Región de Los Lagos, Chile. Revista Geográfica de Valparaíso, 34, 203-218.

Ramírez, C., Sandoval, V., San Martín, C., Álvarez, M., Pérez, Y., \& Novoa, C. (2012). El paisaje rural antropogénico de Aisén, Chile: Estructura y dinámica de la vegetación. Gayana Botánica, 69, 219-231.

Raunkiaer, C. (1934). Life-form of plants and terrestrial plant geography. Oxford: Clarendon Press.

Roig, F. A. (1994). La vegetación de la Patagonia. In M. Correa (Ed.), Flora Patagonica. Buenos Aires: Instituto Nacional de Tecnología Agropecuaria.

Roig, F. A., Anchorena, J., Dollenz, O., Faggi, A. M., \& Mendez, E. (1985). Las comunidades vegetales de la transecta botánica de la Patagonia Austral. In O. Boelcke, D. M. Moore \& F. A. Roig (Eds.), Transecta Botánica de la Patagonia Austral (pp. 350518). Consejo Nacional de Investigaciones Científicas y Técnicas (Buenos Aires), Instituto de la Patagonia (Punta Arenas), Royal Society (Londres).

Rozzi, R., Armesto, J. J., Gutiérrez, J. R., Massardo, F., Likens, G. E., Anderson, C. B., Poole, A.,...Arroyo, M. T. K. (2012). 
Integrating ecology and environmental ethics: earth stewardship in the southern end of Americas. Bioscience, 62, 226-236. Society for Ecological Restoration International Science and Policy Working Group. (2004). The SER International Primer on Ecological Restoration. www.ser. org \& Tucson: Society for Ecological RestorationInternational.

Vidal, O. J. (2005). Flora exótica adyacente a senderos remotos en el Parque Nacional Torres del Paine (Magallanes, Chile). Tesis de Licenciatura en Ciencias Biológicas. Valdivia, Chile: Facultad de Ciencias, Universidad Austral de Chile.

Vidal, O. J. (2007). Flora Torres del Paine field guide. Punta Arenas: Fantástico Sur.

Vidal, O. J. (2012). Anthropogenic disturbances affecting southern beech (Nothofagus pumilio) forests in Torres del Paine Biosphere Reserve, southern Chilean
Patagonia. Tesis de Doctorado. Freiburg, Germany: Faculty of Forests and Environmental Sciences, Albert-Ludwigs University.

Vidal, O. J. (2015). Editorial: Introducción al número especial sobre restauración ecológica en la Ecorregión Magallánica Subantártica. Anales del Instituto de la Patagonia, 43, 7-9.

Wikum, D., \& Shanholtzer, G. F. (1978). Application of the Braun-Blanquet coverabundance scale for vegetation analysis in land development studies. Environmental Management, 2, 323-329.

Wink, M. (2006). Schriftzeichen im Logbuch des lebens: Molekulare Evolutionsforschung. Biologie in unserer Zeit, 36, 26-37.

Zuloaga, F., Morrone, O., \& Belgrano, M. (2008). Catálogo de las plantas vasculares del cono sur. Missouri: Missouri Botanical Garden Press.

\section{Anexo 1}

Caracterización taxonómica y ecológica la flora encontrada en los 35 censos de vegetación levantados en el presente estudio. Grupos (Gru): Dic= dicotiledóneas, Mon= monocotiledóneas); Origen fitogeográfico (Ori): Aut= autóctonas, Alo= Alóctonas; Formas de vida de Raunkiaer (Rau): Fan= fanerófitos, Cam= Caméfitos, Hem= hemicriptófitos, Ter= terófitos, Geo= geófitos; Síndromes de dispersión (Dis): Ext= dispersión externa (exozoócoría); End= dispersión interna (endozoocoría); Aut= dispersión autócora; Vie = dispersión por viento (anemócoras); $\mathrm{Sin}=$ sin atributos morfológicos que favorezcan la dispersión del propágulo; Capacidad de rebrote post-fuego (Res): Reb= con rebrote, Nor= Sin rebrote, ?= Sin información; Tipo de clonación (Clo): Sup= superficial, Sub= clonación subterraánea; Sin= sin clonación vegetativa observada; Fre = Frecuencia absoluta; $\mathrm{Cob}=$ Cobertura relativa; VI= Valor de importancia.

\begin{tabular}{lccccccccccc}
\hline Nombre cientifico & Acr & Gru & Fam & Ori & Rau & Dis & Res & Clo & Fre & Cob & VI \\
\hline Acaena pinnatifida Ruiz \& Pav. & Acpi & Dic & Ros & Aut & Hem & Ext & Reb & Sup & 20 & 0,8 & 4,2 \\
Acaena splendens Hook. \& Arn. & Acsp & Dic & Ros & Aut & Hem & Ext & Reb & Sup & 15 & 0,6 & 3,2 \\
Achillea millefolium L. & Acmi & Dic & Ast & Alo & Hem & Sin & Reb & Sup & 11 & 1,0 & 2,9 \\
Adesmia lotoides Hook. f. & Adlo & Dic & Fab & Aut & Hem & Sin & Nor & Sup & 21 & 1,9 & 5,5 \\
Adesmia pumila Hook. f. & Adpu & Dic & Fab & Aut & Hem & Sin & Nor & Sup & 11 & 0,6 & 2,4 \\
Agrostis capillaris L. & Agca & Mon & Poa & Alo & Hem & Ext & Reb & Sin & 3 & 0,1 & 0,6 \\
Aira caryophyllea L. & Aica & Mon & Poa & Alo & Ter & Sin & Nor & Sup & 14 & 1,5 & 3,8 \\
Aira praecox L. & Aipr & Mon & Poa & Alo & Ter & Sin & Nor & Sup & 7 & 0,4 & 1,6 \\
Alopecurus magellanicus Lam. & Alma & Mon & Poa & Aut & Hem & Aut & Reb & Sin & 1 & 0,1 & 0,2 \\
Alstroemeria patagonica Phil. & Alpa & Mon & Als & Aut & Geo & Aut & Reb & Sub & 11 & 0,4 & 2,3 \\
\hline
\end{tabular}




\begin{tabular}{|c|c|c|c|c|c|c|c|c|c|c|c|}
\hline Nombre científico & Acr & Gru & Fam & Ori & Rau & Dis & Res & Clo & Fre & Cob & VI \\
\hline $\begin{array}{l}\text { Arjona patagonica Hombr. \& Jacq. } \\
\text { ex Decne. }\end{array}$ & Arjpat & Dic & San & Aut & Hem & Sin & Nor & $\operatorname{Sin}$ & 12 & 0,5 & 2,5 \\
\hline Armeria maritima (Mill.) Willd. & Arma & Dic & Plu & Aut & Hem & Sin & Reb & Sin & 19 & 0,7 & 3,9 \\
\hline Astragalus palenae (Phil.) Reiche & Aspa & Dic & Fab & Aut & Cam & Sin & Nor & Sin & 1 & 0,1 & 0,2 \\
\hline Azorella monantha Clos. & Azmo & Dic & Api & Aut & Cam & Sin & Nor & Sin & 6 & 0,8 & 1,8 \\
\hline Baccharis magellanica (Lam.) Pers. & Bama & Dic & Ast & Aut & Cam & Vie & Reb & Sin & 16 & 3,6 & 6,3 \\
\hline Berberis microphylla Lam. & Bemi & Dic & Ber & Aut & Fan & Int & Reb & Sin & 19 & 3,1 & 6,4 \\
\hline $\begin{array}{l}\text { Bolax caespitosa Hombr. \& Jacquinot } \\
\text { ex Decne }\end{array}$ & Boca & Dic & Api & Aut & Cam & Sin & ? & Sin & 1 & 0,1 & 0,2 \\
\hline Bolax gummifera (Lam.) Spreng. & Bogu & Dic & Api & Aut & Cam & Sin & $?$ & Sin & 2 & 0,1 & 0,4 \\
\hline Bromus catharticus Vahl & Brca & Mon & Poa & Alo & Hem & Ext & $?$ & Sin & 4 & 0,1 & 0,8 \\
\hline Calceolaria biflora Lam. & Cabi & Dic & Scr & Aut & Hem & Sin & ? & Sin & 7 & 0,3 & 1,4 \\
\hline Capsella bursa-pastoris (L.) Medicus & Cabu & Dic & Cru & Alo & Ter & Sin & ? & Sin & 2 & 0,3 & 0,6 \\
\hline Carduus nutans L. & Canu & Dic & Ast & Alo & Ter & Vie & $?$ & Sin & 4 & 0,6 & 1,3 \\
\hline Cerastium arvense L. & Cear & Dic & Car & Alo & Hem & Sin & Reb & Sin & 14 & 0,5 & 2,9 \\
\hline Collomia biflora (Ruiz \& Pav.) Brand. & Cobi & Dic & Pol & Aut & Hem & Sin & ? & Sin & 2 & 0,1 & 0,4 \\
\hline $\begin{array}{l}\text { Colobanthus subulatus (d`Urv.) } \\
\text { Hook. f. }\end{array}$ & Cosu & Dic & Car & Aut & Hem & Sin & $?$ & Sin & 1 & 0,1 & 0,2 \\
\hline Conium maculatum $\mathrm{L}$. & Coma & Dic & Api & Alo & Ter & Vie & ? & $\operatorname{Sin}$ & 3 & 0,6 & 1,1 \\
\hline Deschampsia flexuosa (L.) Trin. & Defl & Mon & Poa & Alo & Hem & Ext & Reb & Sup & 9 & 0,3 & 1,9 \\
\hline $\begin{array}{l}\text { Descurainia sophia (L.) Web. ex } \\
\text { Prantl. }\end{array}$ & Deso & Dic & Bra & Alo & Ter & Sin & Reb & Sin & 16 & 0,9 & 3,6 \\
\hline Erodium cicutarium (L.) L`Herit & Erci & Dic & Ger & Alo & Ter & Aut & Reb & Sin & 4 & 0,1 & 0,8 \\
\hline Erophila verna (L.) DC. & Erve & Dic & Bra & Alo & Ter & $\operatorname{Sin}$ & $?$ & Sin & 1 & 0,1 & 0,2 \\
\hline Euphrasia antarctica Benth. & Euan & Dic & Oro & Aut & Hem & Sin & $?$ & Sin & 1 & 0,1 & 0,2 \\
\hline Festuca gracillima Hook. f. & Fegr & Mon & Poa & Aut & Hem & Ext & Reb & Sup & 13 & 2,8 & 5,0 \\
\hline Festuca magellanica Lam. & Fema & Mon & Poa & Aut & Hem & Ext & Reb & Sup & 12 & 0,8 & 2,8 \\
\hline Galium fuegianum Hook. f. & Gafu & Dic & Rub & Aut & Hem & Int & $?$ & Sup & 1 & 0,1 & 0,2 \\
\hline Galium odoratum (L.) Scop & Gaod & Dic & Rub & Aut & Hem & Int & $?$ & Sup & 9 & 0,3 & 1,9 \\
\hline Gamochaeta nivalis Cabrera & Gani & Dic & Ast & Aut & Hem & Vie & $?$ & Sin & 1 & 0,1 & 0,2 \\
\hline Geranium berteroannum Hooker f. & Gebe & Dic & Ros & Aut & Hem & Aut & Reb & Sin & 1 & 0,1 & 0,2 \\
\hline Hieracium antarcticum Speg. & Hian & Dic & Ast & Aut & Hem & Vie & Reb & Sin & 4 & 0,1 & 0,8 \\
\hline Holcus lanatus L. & Hola & Mon & Poa & Alo & Hem & Ext & Reb & Sup & 2 & 0,1 & 0,4 \\
\hline Hordeum comosum J. Presl. & Hoco & Mon & Poa & Alo & Hem & Ext & $?$ & Sin & 4 & 0,1 & 0,8 \\
\hline Hypochoeris radicata L. & Hyra & Dic & Ast & Alo & Hem & Vie & Reb & Sin & 1 & 0,2 & 0,4 \\
\hline Lathyrus nervosus Lam. & Lane & Dic & Fab & Aut & Hem & $\operatorname{Sin}$ & Sin & Sup & 1 & 0,1 & 0,2 \\
\hline Luzula alopecurus Desv. & Lual & Mon & Jun & Aut & Hem & Sin & Reb & Sub & 14 & 0,5 & 2,9 \\
\hline
\end{tabular}




\begin{tabular}{|c|c|c|c|c|c|c|c|c|c|c|c|}
\hline Nombre científico & Acr & Gru & Fam & Ori & Rau & Dis & Res & Clo & Fre & Cob & VI \\
\hline $\begin{array}{l}\text { Microsteris gracilis (Douglas ex } \\
\text { Hooker) Greene }\end{array}$ & Migr & Dic & Pol & Aut & Ter & Sin & Reb & Sin & 20 & 0,8 & 4,2 \\
\hline $\begin{array}{l}\text { Mulgaraea tridens (Lag.) N. O'Leary } \\
\& \text { P. Peralta }\end{array}$ & Mutr & Dic & Ver & Aut & Fam & Sin & Reb & Sin & 35 & 48,9 & 54,9 \\
\hline Mulinum spinosum (Cav.) Pers. & Musp & Dic & Api & Aut & Cam & Sin & Reb & Sin & 33 & 11,3 & 16,9 \\
\hline $\begin{array}{l}\text { Myosotis arvensis Link ex Roemer \& } \\
\text { Schultes }\end{array}$ & Myar & Dic & Bor & Alo & Ter & Sin & Sin & Sin & 1 & 0,1 & 0,2 \\
\hline $\begin{array}{l}\text { Myosotis stricta Link. ex Roem \& } \\
\text { Schult. }\end{array}$ & Myst & Dic & Bor & Alo & Ter & Sin & $?$ & Sin & 4 & 0,3 & 1,0 \\
\hline $\begin{array}{l}\text { Nassauvia abbreviata (Hook. \& Arn.) } \\
\text { Dusén }\end{array}$ & Naac & Dic & Ast & Aut & Cam & Vie & ? & Sin & 15 & 0,6 & 3,1 \\
\hline Oenothera stricta Ledeb, ex Link. & Oest & Dic & Ona & Alo & Hem & $\operatorname{Sin}$ & ? & $\operatorname{Sin}$ & 2 & 0,4 & 0,7 \\
\hline $\begin{array}{l}\text { Olsinium biflora (Thunb.) R. C. } \\
\text { Forster }\end{array}$ & Olbi & Mon & Iri & Aut & Geo & Sin & ? & Sub & 2 & 0,1 & 0,4 \\
\hline Oxalis enneaphylla Cav. & Oxen & Dic & Oxa & Aut & Geo & $\operatorname{Sin}$ & ? & Sub & 3 & 0,1 & 0,6 \\
\hline $\begin{array}{l}\text { Petrorhagia dubia (Raf.) G. López \& } \\
\text { Romo }\end{array}$ & Pedu & Dic & Car & Aut & Ter & Sin & ? & $\operatorname{Sin}$ & 16 & 0,8 & 3,6 \\
\hline Phacelia secunda (J. F.) Gmelin & Phse & Dic & Hyd & Aut & Hem & Vie & $\operatorname{Sin}$ & $\operatorname{Sin}$ & 1 & 0,1 & 0,2 \\
\hline Plantago lanceolata L. & Plla & Dic & $\mathrm{Pla}$ & Alo & Hem & Sin & Reb & $\operatorname{Sin}$ & 18 & 1,5 & 4,5 \\
\hline Poa annua L. & Poan & Mon & Poa & Alo & Ter & $\operatorname{Sin}$ & ? & $\operatorname{Sin}$ & 1 & 0,1 & 0,2 \\
\hline Polygala darwiniana A. W. Benn. & Poda & Dic & Pol & Aut & Hem & $\operatorname{Sin}$ & ? & $\operatorname{Sin}$ & 9 & 0,3 & 1,9 \\
\hline Rumex acetosella L. & Ruac & Dic & Pol & Alo & Hem & Sin & Reb & Sub & 15 & 1,0 & 3,6 \\
\hline $\begin{array}{l}\text { Rytidosperma virescens (É. Desv.) } \\
\text { Nicora }\end{array}$ & Ryvi & Mon & Poa & Aut & Hem & Ext & ? & $\operatorname{Sin}$ & 16 & 0,6 & 3,4 \\
\hline Senecio patagonicus Hooker \& Arn. & Sepa & Dic & Ast & Aut & Fan & Vie & Reb & Sin & 22 & 4,3 & 8,0 \\
\hline $\begin{array}{l}\text { Sisirhynchium arenarium Phil. ex } \\
\text { Baker }\end{array}$ & Siar & Mon & Iri & Aut & Geo & Sin & ? & Sub & 17 & 0,7 & 3,5 \\
\hline Sonchus asper (L.) Hill. & Soas & Dic & Ast & Alo & Ter & Vie & ? & Sub & 2 & 0,2 & 0,6 \\
\hline Taraxacum gilliesii Hook. \& Arn. & Tagi & Dic & Ast & Aut & Hem & Vie & Reb & Sub & 9 & 0,7 & 2,1 \\
\hline Taraxacum officinale Weber & Taof & Dic & Ast & Alo & Hem & Vie & Reb & Sub & 2 & 0,2 & 0,6 \\
\hline Trifolium repens L. & Trre & Dic & Fab & Alo & Hem & Sin & Reb & Sub & 9 & 0,7 & 2,1 \\
\hline $\begin{array}{l}\text { Tripleurospermum maritimum (L.) } \\
\text { W.D. J. Koch }\end{array}$ & Trma & Dic & Ast & Alo & Ter & Sin & ? & Sup & 2 & 0,4 & 0,7 \\
\hline Vicia magellanica Hooker f. & Vima & Dic & $\mathrm{Fab}$ & Aut & Hem & $\operatorname{Sin}$ & Reb & Sup & 7 & 0,6 & 1,8 \\
\hline Viola maculata Cav. & Voma & Dic & Vio & Aut & Hem & $\operatorname{Sin}$ & Reb & $\operatorname{Sin}$ & 8 & 0,5 & 1,8 \\
\hline
\end{tabular}


O. VIDAL et al. 